\title{
Was kosten Studienvisa? Eine global vergleichende Analyse
}

\section{ZUSAMMENFASSUNG}

Für internationale Studierendenmobilität ist oft ein Visum nötig. Die Kosten dafür variieren enorm, wie Daten des Global Mobilities Project (GMP) zeigen. So zahlen Studierende aus dem Globalen Süden teils mehr als das Doppelte als Studierende aus Europa. Noch größer sind die Unterschiede, wenn man das Wohlstandsniveau berücksichtigt: Während die Kosten für Studierende aus Westeuropa im Schnitt nur dem Bruchteil eines durchschnittlichen Tageseinkommens entsprechen, müsste man z. B. in Zentralasien fast drei, in Subsahara-Afrika sogar mehr als fünf Wochen arbeiten, um sich ein Visum leisten zu können. Es ergibt sich insgesamt also eine paradoxe Situation: Studierende aus reicheren Ländern müssen weniger bezahlen, wenn sie international mobil sein wollen. Diese globalen Ungleichheiten berühren bedeutsame Aspekte individueller Ungerechtigkeit und weisen auf die Dysfunktionalität von Visaregimes, die internationale Mobilität talentierter Menschen aus dem Globalen Süden strukturell einschränken.

\section{Einleitung}

Internationale Studierendenmobilität ist global gesehen von großer Ungleichheit geprägt. Ein Großteil der Mobilität findet zwischen einer kleinen Anzahl von Länderpaaren statt (Delhey et al., 2020, S. 112 ff.; Deutschmann, 2021, S. 175). Die Hälfte aller international mobilen Studierenden weltweit entfällt auf gerade einmal sieben Gastländer. ${ }^{1}$ Allein das wirft wichtige Fragen nach Chancengleichheit und globaler Gerechtigkeit auf. Doch es gibt eine weitere Dimension, die bisher relativ wenig beachtet wurde: den Preis, den international mobile Studierende zahlen müssen, um ein Visum für ihren geplanten Auslandsaufenthalt zu beantragen. Dass auch dieser Faktor je nach Herkunftsland stark divergiert, wird in dieser Arbeit thematisiert.

Das Festlegen von Visarestriktionen ist für Staaten ein wichtiges Mittel zur Regulierung eingehender Mobilität aus dem Ausland. Ob ein Visum verlangt wird (und welche Kriterien gegebenenfalls dessen Genehmigung zugrunde gelegt werden) ist damit

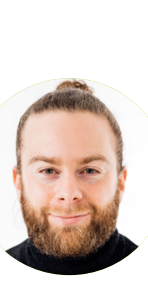

\section{AUTOR}

\section{Dr. Emanuel Deutschmann ist} Juniorprofessor für Soziologische Theorie an der Europa-Universität Flensburg und Associate am Migration Policy Centre des Europäischen Hochschulinstituts in Florenz. Kontakt: emanuel.deutschmann@uni-flensburg.de 
eine wesentliche Stellschraube nationalstaatlicher Grenzkontrolle. Staaten haben durch Visa die Möglichkeit, potenzielle Einreisen (a) im Vorhinein und (b) weit weg vom eigenen Territorium - typischerweise in Konsulaten im Herkunftsland der Reisewilligen - zu prüfen. Sie ermöglichen es also, vorzuselektieren und unerwünschte Personen bereits vor Reiseantritt abzuhalten.

Czaika et al. (2018) zeigen, dass historische und geopolitische Beziehungen eine Rolle dabei spielen, ob und zu welchen Konditionen Staaten von Angehörigen anderer Staaten Visa verlangen. Neben bilateralen Vereinbarungen sind es vor allem Staatenverbünde wie die Europäische Union, die Westafrikanische Wirtschaftsgemeinschaft (ECOWAS) oder der Verband Ostasiatischer Nationen (ASEAN), die Vereinbarungen für Visabefreiungen (engl. visa waivers) treffen. Insgesamt wird jedoch nach wie vor zwischen etwa drei Vierteln aller Länderpaare weltweit ein Visum verlangt
Visa, also Reisegenehmigungen, weltweit kostet abhängig davon, woher man kommt und in welches Land man geht. Gesammelt wurden Daten in sechs Kategorien: Tourismus, Geschäftsreisen, Arbeit, Familienzusammenführung, Transit - und eben Studierendenmobilität. ${ }^{3}$

\section{Methodisches Vorgehen}

Die Daten des GMP Global Visa Cost Dataset wurden von April bis September 2019 gesammelt, einige Lücken im November 2020 durch eine zusätzliche Datenerhebung geschlossen. Quellen waren in den allermeisten Fällen die offiziellen staatlichen Internetseiten des Ziellands, auf denen Informationen zu den Visakosten je nach Herkunftsland zu finden sind. Dabei kann es sich um die Webseite eines Ministeriums oder auch eines Konsulats handeln. Alle Visakosten beziehen sich auf das Jahr 2019 und sind in US-Dollar konvertiert. Angegeben sind die Kosten für die Beantragung eines Visums, in diesem Fall für einen Studienaufenthalt in einem bestimmten Land, abhängig davon, aus welchem Land man kommt.

Erfolgsaussichten bzw. Ablehnungsquoten und damit im Zusammenhang stehend z. B. der Umstand, dass im Falle eines abgelehnten Visumsantrags meist Geld verloren geht und zudem der Auslandsaufenthalt nicht möglich ist, wurden bei der Datensammlung nicht einbezogen. Genauso wenig berücksichtigt wurden zusätzliche Kostenfaktoren und andere Hindernisse, wie etwa die Ausstellung eines Reisepasses, der Weg zum Konsulat oder der Nachweis finanzieller Rücklagen. Es handelt sich bei den erhobenen Visakosten also eher um das absolute Minimum an finanziellem Aufwand; die realen Hürden und tatsächlichen Ausgaben können noch deutlich größer sein. Insgesamt enthält der Datensatz Informationen über die Kosten von knapp 100.000 Visa. In der Kategorie „Studierendenmobilität" sind die Kosten von Einreiseerlaubnissen für 7.454 Länderpaarkombinationen erfasst.

Einschränkend ist zu sagen, dass die Daten des GMP Global Visa Cost Dataset (frei verfügbar auf der Website des Projekts) in der Kategorie in Florenz angesiedelten Global Mobilities Project

2 Genauere Informationen zum Global Mobilities Project finden sich auf der Projektwebsite: https://migrationpolicycentre.eu/projects/globalmobilities-project.

3 Befunde basierend auf den Daten für Tourismusvisa wurden bereits publiziert (Recchi et al., 2021). 
„Studierendenmobilität“ deutlich unvollständiger sind als beispielsweise in der Kategorie „Tourismus“. Dies hat zum Teil substanzielle Gründe (zwischen vielen Länderpaaren weltweit bewegen sich schlicht keine Studierenden; siehe die eingangs erwähnten Ungleichheitsbefunde), zum Teil liegt es aber auch an der schlechteren Zugänglichkeit der entsprechenden Informationen. Es ist wichtig, diese Limitierung im Hinterkopf zu behalten, wenn man auf die folgenden Befunde blickt. Nichtsdestotrotz erlauben die Daten einen ersten Einblick in die globalen Ungleichheiten bei Visakosten für Studierende.

\section{Zentrale Befunde}

Studierende aus dem Globalen Süden müssen deutlich mehr für Visa bezahlen als Studierende aus dem Globalen Norden. Einen ersten Eindruck hiervon vermittelt Abbildung 1. Sie zeigt, wie hoch die Kosten für ein Studienvisum im Durchschnitt je nach Herkunftsregion sind, angegeben in US-Dollar.

Wie man sieht, ist die Spannweite groß: Während Studierende aus Westeuropa im Schnitt knapp 43 US-Dollar für ein Studienvisum bezahlen, müssen Studierende aus Süd- und Zentralasien mit etwas über 100 US-Dollar mehr als das Doppelte ausgeben.

ABB. 1. DURCHSCHNITTLICHE ANTRAGSKOSTEN FÜR STUDIENVISA NACH HERKUNFTSREGION

(IN US-DOLLAR)

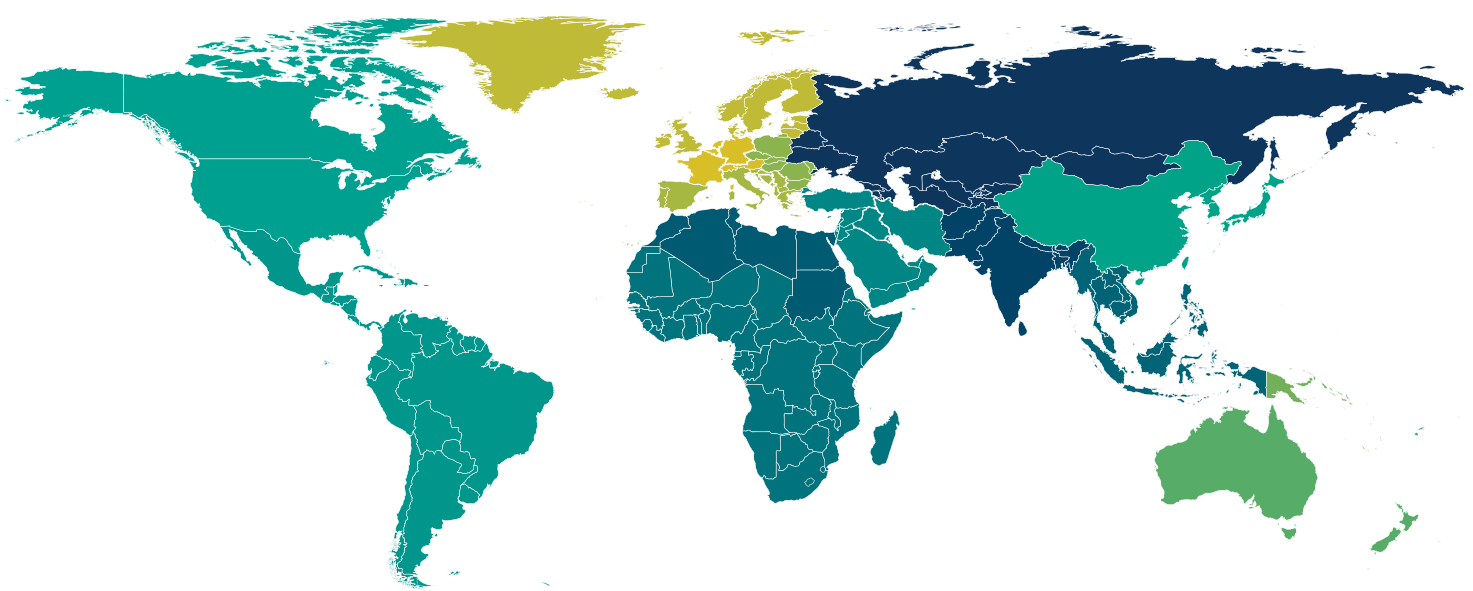

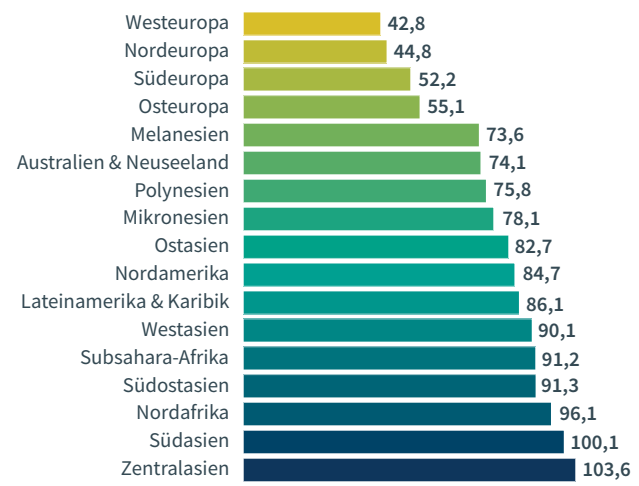

Was passiert nun, wenn auch das in unterschiedlichen Teilen der Welt unterschiedliche Verdienstniveau berücksichtigt wird? Dies zeigt Abbildung 2, in der die Studienvisakosten nach Herkunftsregion in durchschnittlichen Tageseinkommen dargestellt sind. In anderen Worten: Wie lange muss jemand aus der jeweiligen Region im Schnitt arbeiten, um sich ein Studienvisum leisten zu können?
Wie man sieht, ist die globale Diskrepanz noch einmal deutlich größer geworden. Während Menschen in West- und Nordeuropa, Nordamerika, Australien und Neuseeland im Schnitt nur den Bruchteil eines Tags arbeiten müssen, um sich ein Visum leisten zu können, sind es in Zentral- und Südasien mehrere Wochen und in Subsahara-Afrika sogar mehr als ein Monat. Es gibt also enorme globale Unterschiede. Und für einzelne Herkunftsländer können die Kosten nochmal deutlich höher liegen als für die hier gezeigten aggregierten Herkunftsregionen (vgl. Recchi et al., 2021). 


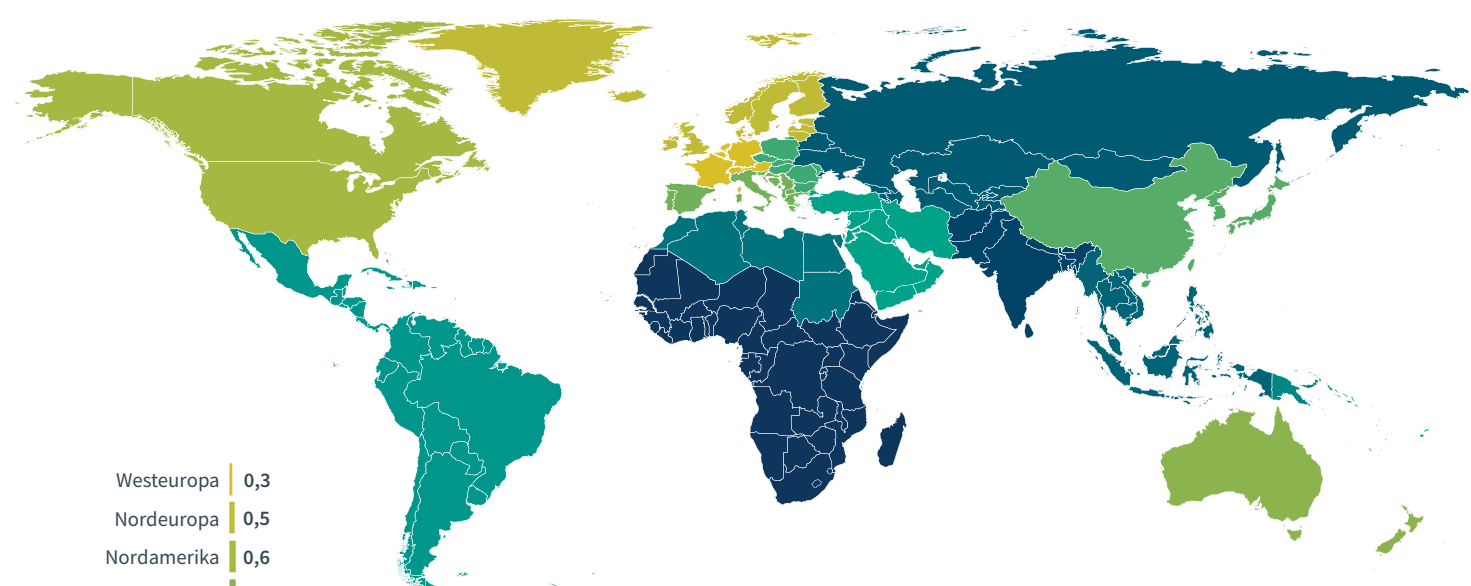

Australien \& Neuseeland $\mid 0,6$ Südeuropa $\quad 2,2$ Ostasien $\quad 2,9$ Osteuropa $\quad 3,6$ Mikronesien $\quad 5,7$ Westasien 6,0 Polynesien $\quad 6,1$ Lateinamerika \& Karibik $\quad 6,3$

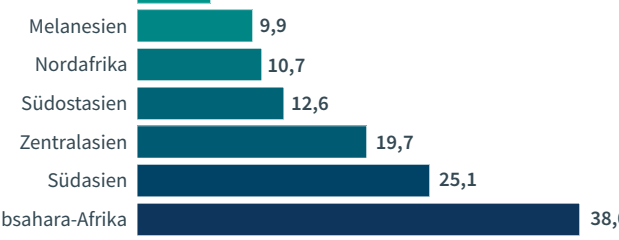

Insgesamt ergibt sich eine paradoxe Situation: Je reicher ein Herkunftsland (gemessen z. B. am Bruttonationaleinkommen pro Kopf), desto weniger müssen Studierende, die in ihm leben, bezahlen, um für einen Studienaufenthalt in ein anderes Land gehen zu können, und zwar sowohl absolut betrachtet (in US-Dollar) wie relativ zum Durchschnittseinkommen.

Im Matthäus-Evangelium der Bibel heißt es: „Denn wer da hat, dem wird gegeben, dass er die Fülle habe; wer aber nicht hat, dem wird auch das genommen, was er hat." Der Soziologe Robert K. Merton hat daraus in den 1960er Jahren den MatthäusEffekt der Wissenschaft abgeleitet. Er zeigte, dass bekannte Forschende mehr zitiert werden, schlicht weil sie bekannt sind (Merton, 1968). ${ }^{4}$ Bei den Einreiseerlaubnissen liegt ein ganz ähnliches Phänomen vor, das man als den Matthäus-Effekt der Visakosten bezeichnen könnte: Wer aus einem reicheren
Land kommt, muss weniger bezahlen, bleibt also reich, während Studierende aus ärmeren Ländern mehr ausgeben müssen und so tendenziell noch ärmer werden.

Da höhere Visakosten auch Studienaufenthalte verhindern können (und zusätzliche Hindernisse wie höhere Ablehnungsquoten von Anträgen sind hier noch gar nicht berücksichtigt), kommt es möglicherweise zu einer selbstverstärkenden Eigendynamik. Denn wer aufgrund prohibitiv hoher Kosten einen Studienaufenthalt im Ausland nicht antreten kann, wird ebenfalls nicht von den zu erwartenden positiven Effekten profitieren, etwa in Form von besseren Jobs, Aufstiegsmöglichkeiten und höheren Einkommen, die eine solche Auslandserfahrung auf dem Arbeitsmarkt bringen kann (Gerhards et al., 2017; Netz et al., 2020). Somit stabilisiert sich die globale Ungleichheit also tendenziell selbst.

Bisher haben wir vor allem darauf geblickt, wer wie viel bezahlen muss. Nun können wir zusätzlich einbeziehen, wer wie viel für ein Studienvisum verlangt. Dies ist in Abbildung 3 für die einzelnen Weltregionen dargestellt. Je größer der Kreis, desto mehr fordert eine Weltregion im Schnitt für Studienvisa. Ist der Kreis blau, bedeutet dies, dass Studierende aus dieser Weltregion selbst wenig bezahlen müssen, wenn sie ein Studienvisum benötigen - ist er rot, müssen sie hingegen relativ viel ausgeben. Die Pfeile zwischen den Kreisen zeigen die Reiserichtung an. Die Dicke und Farbgebung des Pfeils

4 Merton tat dies unter Zuhilfenahme von Forschungsmaterial seiner Frau Harriet Zuckerman, die nicht als Koautorin genannt wurde und sehr viel weniger berühmt geblieben ist als er (vgl. Merton 1995). 
entspricht dabei der Höhe der durchschnittlichen Kosten eines Studienvisums.

Auch hier werden diskrepante Muster erkennbar: Europa muss relativ wenig bezahlen, verlangt aber selbst ebenso vergleichsweise wenig. Nordamerika hingegen muss zwar gleichfalls verhältnismäßig wenig ausgeben (mit Ausnahme der im Vergleich zu anderen teuren Studierendenmobilität in Richtung Lateinamerika), fordert aber sehr viel von einreisenden Studierenden (man beachte die roten, außergewöhnlich dicken eingehenden Pfeile aus allen anderen Weltregionen). Afrika und Asien wiederum sind Regionen, die relativ wenig verlangen, aber selbst sehr viel bezahlen müssen. Man sieht außerdem, dass die Studienvisakosten in die gegenläufigen Richtungen oft sehr unterschiedlich sind (z. B. sehr hoch von Asien nach Nordamerika, aber sehr niedrig von Nordamerika nach Asien). Das globale Netzwerk der Studienvisakosten scheint also insgesamt eher von Ungleichgewichten geprägt als von Rezi-
Wichtig ist hier ebenfalls noch einmal der Hinweis, dass der Datensatz teilweise lückenhaft ist und daher nur ein ungefähres Bild der globalen Netzwerkbeziehungen gezeichnet werden kann (aus diesem Grund fehlt auch Ozeanien in der Darstellung). Außerdem verdeckt die hier gezeigte Makroperspektive die Varianz innerhalb der Weltregionen. Es empfiehlt sich zusätzlich der Blick in den frei verfügbaren Datensatz, der alle Visakosten nach Länderpaaren aufschlüsselt. Nichtsdestotrotz verdeutlicht die Grafik, dass das globale Netzwerk der Studienvisakosten von großer Ungleichheit geprägt ist.

\section{Fazit \& Diskussion}

Reisegenehmigungen erlauben es Staaten, eingehende Mobilität zu regulieren (Mau, 2013; Czaika \& Neumayer, 2017). Für potenzielle Reisende sind Visa jedoch mit Kosten verbunden und stellen eventuell eine Hürde für internationale Mobilität dar. Sind die Kosten prohibitiv hoch, können sie Auslandsaufenthalte verhindern. Damit nehmen Visakosten Einfluss auf die globale Struktur internationaler Studierendenmobilität.

Zur genaueren Untersuchung wurden in diesem Beitrag weltweit vergleichend die Kosten von Studienvisa betrachtet. Dabei zeigte sich, dass die Kosten sehr ungleich ausfallen, je nachdem woher man kommt und wohin man geht. So müssen Menschen aus Ländern des Globalen Südens oft ein Vielfaches dessen ausgeben, was Menschen aus dem Globalen Norden, insbesondere aus West- und Nordeuropa, für Studienvisa zu bezahlen haben. Noch größer wird diese Diskrepanz, wenn man das Wohlstandsniveau einbezieht: Während man in Westeuropa, Skandinavien, Nordamerika, Australien und Neuseeland im Schnitt nur den Bruchteil eines Tags arbeiten müsste, um sich ein Visum für einen Studienaufenthalt im Ausland leisten zu können, entsprechen die Kosten für eine Einreiseerlaubnis in weiten Teilen des Globalen Südens mehreren Wochen durchschnittlicher Arbeitszeit.

Dieses Ungleichgewicht wirft wichtige Fragen auf. Aus normativer Sicht kann nach der Gerechtigkeit dieses Systems gefragt werden: Warum sollte die regionale Herkunft Einfluss darauf haben, wie viel 
jemand für das Visum für einen Studienaufenthalt bezahlen muss? Sollte nicht jede Person - ganz unabhängig von ihrem Geburtsort oder ihrer Nationalität - dieselben Chancen auf einen Studienaufenthalt im Ausland haben? Wie bereits erwähnt, führen solche Auslandsaufenthalte zu „transnationalem Humankapital" (Gerhards et al., 2017), das wiederum materielle Vorteile auf dem Arbeitsmarkt bringen kann. Ist der Zugang selektiv, stellt dies ein Gerechtigkeitsproblem dar.

Aus funktionalistischer Perspektive lässt sich die Frage stellen, ob das jetzige System nicht dazu führt, dass das Talent vieler junger Menschen ungenutzt bleibt. Wenn die Kosten für die Beantragung eines Visums dem Durchschnittsgehalt mehrerer Wochen oder gar Monate entsprechen, bedeutet dies für weite Teile der Bevölkerung ein unüberwindbares Hindernis. Das gilt umso mehr, als in der hier präsentierten Analyse zusätzlich anfallende Hemmnisse noch gar nicht berücksichtigt wurden, etwa global
Eine Lösung dieses Problems bestünde darin, die Kosten für die Beantragung von Visa abhängig vom Wohlstandsniveau des Herkunftslands zu gestalten: je niedriger der Lebensstandard, desto geringer die Visakosten - also genau gegenteilig zum jetzigen System mit seinem paradoxen Matthäus-Effekt. Eine andere Lösung wäre, die Kosten für Studienvisa generell abzusenken oder ganz abzuschaffen. Da internationale Studierende global gesehen im Vergleich zu touristisch und geschäftlich Reisenden eine sehr kleine Gruppe darstellen, spricht wenig dagegen, Visakosten für sie so gering wie möglich zu halten.

Die Beseitigung von Visarestriktionen führt zudem nicht nur zu mehr, sondern auch zu besseren Bewerbungen internationaler Studierender (Chevalier, 2014). Und Daten aus Dänemark zeigen, dass selbst dort, wo internationale Studierende weitreichende staatliche Förderung erhalten, das Aufnahmeland auch finanziell von internationalen Studierenden profitiert (Smith, 2015). Das Senken von Visakosten könnte daher ein geeignetes Mittel für die Beseitigung einer Form globaler Ungleichheit sein, aus der viele Seiten einen Nutzen ziehen würden. laubnissen, bürokratische, räumliche und technische in ländlichen Gebieten oder die Nachweispflicht für teils sehr hohe finanzielle Rücklagen (z. B. in Form von Sperrkonten). Diese stellen oft noch größere Hürden dar als die Visakosten selbst.

Zusammengefasst: Ein wirklich meritokratisches System wäre daran interessiert, den talentiertesten internationalen Studierenden weltweit einen Studienaufenthalt zu ermöglichen - egal, woher sie kommen. Der jetzige Zustand verhindert aber tendenziell internationale Mobilität von hochtalentierten jungen Menschen aus dem Globalen Süden durch teilweise prohibitiv hohe Visakosten. Dies ist nicht nur ungerecht (hinsichtlich der Lebens- und Bildungschancen dieser Menschen), sondern auch dysfunktional (mit Blick auf das Humankapital und das Innovationspotenzial der Gesellschaft). 


\section{DATENZUGANG}

Die Daten des Global Mobilities Project, auf denen die hier präsentierten Befunde beruhen, können unter folgendem Link abgerufen werden: https://zenodo.org/ record/4572529.

\section{LITERATUR}

Chevalier, A. (2014). How to attract foreign students. IZA World of Labor, 36, https://doi.org/10.15185/izawol.36.

Czaika, M., \& Neumayer, E. (2017). Visa restrictions and economic globalisation. Applied Geography, 84, 75-82, https://doi.org/10.1016/j.apgeog.2017.04.011.

Czaika, M., de Haas, H., \& Villares-Varela, M. (2018). The Global Evolution of Travel Visa Regimes. Population and Development Review, 44(3), 589-622, https://doi. org/10.1111/padr.12166.

Delhey, J., Deutschmann, E., Verbalyte, M., \& Aplowski, A. (2020). Netzwerk Europa: Wie ein Kontinent durch Mobilität und Kommunikation zusammenwächst. Wiesbaden: springer VS.

Deutschmann, E. (2021). Mapping the Transnational World: How We Move and Communicate Across Borders, and Why It Matters. Princeton, NJ: Princeton University Press.

Gerhards, J., Hans, S., \& Carlson, S. (2017). Social Class and Transnational Human Capital. How Upper and Middle Class Parents Prepare Their Children for Globalization. London: Routledge
Mau, S. (2013). Das globale Mobilitätsregime und die Ungleichheitsfrage. In H.-G. Soeffner (Hrsg.), Transnationale Vergesellschaftungen. Verhandlungen des 35. Kongresses der Deutschen Gesellschaft für Soziologie in Frankfurt am Main 2010 (S. 467-482). Wiesbaden: Springer VS, https://doi.org/10.1007/978-3-531-18971-0_48.

Merton, R. K. (1968). The Matthew Effect in Science: The reward and communication systems of science are considered. Science, 159(3810), 56-63, https://doi. org/10.1126/science.159.3810.56.

Merton, R. K. (1995). The Thomas Theorem and The Matthew Effect. Social Forces, 74(2), 379-422, https://doi. org/10.2307/2580486.

Netz, N., Hampel, S., \& Aman, V. (2020). What effects does international mobility have on scientists' careers? A systematic review. Research Evaluation, 29(3), 327-351, https://doi.org/10.1093/reseval/rvaa007.

Recchi, E., Deutschmann, E., Gabrielli, L., \& Kholmatova, N. (2021). The global visa cost divide: How and why the price for travel permits varies worldwide. Political Geography, 86, 1-14, https://doi.org/10.1016/j.polgeo.2021.102350.

Smith, B. (2015). Foreign students add \$23.8m to Danish economy despite state funding. The Pie News. Abgerufen am 29.09.2021 von https://thepienews.com/news/foreignstudents-add-23-8m-danish-economy-despite-statefunding.

\section{IMPRESSUM}

\section{Herausgeber}

Deutscher Akademischer Austauschdienst e.V.

German Academic Exchange Service

Kennedyallee 50, D - 53175 Bonn

www.daad.de/de/impressum

Referat Forschung und Studien - S15

\section{Ansprechpartner}

Dr. Jan Kercher

kercher@daad.de
Januar 2022

Als digitale Publikation im Internet veröffentlicht

1. Fassung vom 10.01.2022

(c) DAAD - Alle Rechte vorbehalten

Dieses Werk steht unter der Creative Commons Namensnennung - Nicht kommerziell - Weitergabe unter gleichen Bedingungen 3.0 Deutschland Lizenz (CC BY-NC-SA 3.0 DE) https://creativecommons.org/licenses/by-nc-sa/3.0/de/ 